\section{ISOFORMS OF NEUROPILIN-2 REGULATE DISTINCT MACROPHAGE FUNCTIONS AND ARE ASSOCIATED WITH UNIQUE TUMOR-ASSOCIATED MACROPHAGES IN MURINE AND HUMAN BREAST CANCER}

${ }^{1}$ Rajeev Dhupar, ${ }^{1}$ Katherine Jones, ${ }^{1}$ Amy Powers, ${ }^{1}$ Seth Eisenberg, ${ }^{2}$ Kai Ding, ${ }^{2}$ Fangyuan Chen, ${ }^{3}$ Cecile Nasarre, ${ }^{3}$ Amanda LaRue, ${ }^{4}$ Elizabeth Yeh, 'James Luketich, ${ }^{2}$ Adrian Lee, ${ }^{2}$ Steffi Oesterreich, ${ }^{1}$ Michael Lotze, ${ }^{3}$ Robert Gemmill, ${ }^{1}$ Adam Soloff* ${ }^{1}$ University of Pittsburgh School of Medicine, Pittsburgh, PA, United States; ${ }^{2}$ Womens Cancer Research Center, Pittsburgh, PA, United States; ${ }^{3}$ Medical University of South Carolina, Charleston, SC, United States; ${ }^{4}$ Indiana University School of Medicine, Indianapolis, IN, United States

Background Tumor-associated macrophages (TAMs) exert profound influence over breast cancer progression, promoting immunosuppression, angiogenesis, and metastasis. ${ }^{1}$ Neuropilin2 (NRP2), consisting of NRP2a and NRP2b isoforms, is a coreceptor for heparin-binding growth factors including VEGF-C and Class 3 Semaphorins. Selective upregulation in response to environmental stimuli and independent signaling pathways endow the NRP2 isoforms with unique functionality. ${ }^{2} 3$ We have shown that the two isoforms of NRP2 endow opposing functionality to tumor cells due to distinct signaling pathways, with NRP2b promoting metastatic behavior. ${ }^{3}$ Although NRPs have been shown to regulate macrophage/TAM biology, the role of NRP2 isoforms in TAM functionality has yet to be evaluated.

Methods To assess the contribution of NRP2 isoforms to macrophage biology, conditional NRP2a and NRP2b knockout mice and stable shRNA knockdown of NRP2a or NRP2b in Raw264.7 macrophages were generated. Phagocytosis, lysosomal processing of phagocytosed cargo, cytokine production, and influence on tumor cell migration were assessed in vitro using NRP2 isoform knockdown macrophages. NRP2 isoform expression was evaluated on TAMs from murine 4T1 and EO771 mammary carcinoma models using spectral cytometry and single-cell qPCR. NRP2 isoforms and approximated immune composition were evaluated in paired primary tumors and distant metastasis using RNAseq in a cohort of 99 breast cancer patients. High-dimensional myeloid phenotyping was performed on malignant pleural effusions (MPEs) from breast cancer patients or effusions of benign origin using 33-color spectral cytometry and unbiased computational analysis.

Results NRP2 isoform expression was significantly increased in TAMs from murine tumors compared to macrophages from healthy mammary glands. NRP2 isoforms in human primary and metastatic breast cancer were strongly correlated with one another and positively correlated with increased TAMs. Distinct phenotypes of NRP2 isoform-expressing TAMs in were present in 4T1 and EO771 mouse breast cancers and within MPEs from breast cancer patients which were associated with high levels of activation and potential response to a hypoxic tumor niche. Genetic depletion of either NRP2 isoform resulted in dramatic reduction of LPS-induced IL-10 production, defects in phagosomal processing of apoptotic breast cancer cells, and increase in cancer cell migration following coculture. By contrast, inhibition of IL-6 production was specific in NRP2b knockdown cells while phagocytic uptake of labeled particulates was inhibited only by NPR2a knockdown.

Conclusions These results demonstrate that NRP2 isoforms regulate both shared and distinct functionality in macrophages and that NRP2 isoform expression identifies unique TAM subsets in breast cancer.

Acknowledgements This work was supported by awards from the Susan G. Komen Foundation (CCR15329745), U.S.
Department of Defense (W81XWH1910650), and American Lung Association/Thoracic Surgery Foundation to ACS. RD was supported by funding from a Department of Veteran's Affairs Career Development Award (CX001771-01A2) and the University of Pittsburgh's Dean Faculty Advancement Award. ESY was supported by the NCI of the NIH under R03 CA245774. MTL was supported by the NCI of the NIH under awards R01CA181450 and R01CA206012 as well as ITTC/UPMCE. RD and ACS were further supported by funding from the Department of Cardiothoracic Surgery.

\section{REFERENCES}

1. Williams $C B$, Yeh ES, Soloff AC. Tumor-associated macrophages: unwitting accomplices in breast cancer malignancy. Npj Breast Cancer [Internet]. Breast Cancer Research Foundation/Macmillan Publishers Limited; 2016;2:15025. Available from: http://dx.doi.org/10.1038/npjbcancer.2015.252.

2. Nasarre P, Gemmill RM, Potiron VA, Roche J, Lu X, Barón AE, et al. Neuropilin-2 is upregulated in lung cancer cells during TGF- $\beta 1$-Induced epithelial-mesenchymal transition. Cancer Res [Internet] 2013:73:7111 LP-7121. Available from: http://cancerres.aacrjournals.org/content/73/23/7111.abstract3.

3. Gemmill RM, Nasarre $P$, Nair-Menon J, Cappuzzo $F$, Landi L, D'Incecco $A$, et at. The neuropilin 2 isoform NRP2b uniquely supports TGF $\beta$-mediated progression in lung cancer. Sci Signal [Internet] 2017;10. Available from: http://stke.sciencemag. org/content/10/462/eaag0528.abstract

Ethics Approval The study was approved by the University of Pittsburgh's Institutional Review Board approval number CR19120172-005.

http://dx.doi.org/10.1136/jitc-2021-SITC2021.680 